$01 ; 13.2$

\title{
Модель поверхностного димера в задаче об адсорбции
}

\author{
() С.Ю. Давыдов ${ }^{1}$, А.В. Зубов ${ }^{2}$, А.А. Лебедев ${ }^{1}$ \\ ${ }^{1}$ Физико-технический институт им. А.Ф. Иофффе РАН, Санкт-Петербург, Россия \\ ${ }^{2}$ Санкт-Петербургский национальный исследовательский университет информационных технологий, механики и оптики \\ (Университет ИТМО), Санкт-Петербург, Россия \\ E-mail: sergei_davydov@mail.ru
}

Поступило в Редакцию 7 февраля 2019 г.

В окончательной редакции 11 февраля 2019 г.

Принято к публикации 13 февраля 2019 г.

Адсорбционная система представлена в виде двух областей: димера, включающего адчастицу и поверхностный атом подложки, и всего остального пространства. В качестве подложки рассмотрены металл, полупроводник и графен (полуметалл). Обсуждаются сходства и различия значений чисел заполнения адчастицы, полученных в рамках предлагаемой и стандартной моделей адсорбции.

DOI: 10.21883/PJTF.2019.09.47713.17730

Несмотря на долгую историю, интерес к фундаментальным проблемам адсорбции по-прежнему не спадает (см., например, материалы International conference on the fundamentals of adsorption (FOA), которая в этом году проводится уже в тринадцатый раз, а также обзоры [1-3]). Следует подчеркнуть, что с появлением графеновой тематики интерес к адсорбционным процессам на углеродных структурах значительно возрос в связи с задачей функционализации графена и его использования в сенсорах $[4,5]$.

Обращаясь к современной теории адсорбции, основанной на модельном рассмотрении задачи [6,7], следует отметить два основных подхода: 1) адатом по отношению к подложке рассматривается как примесь, находящаяся на ее поверхности (твердотельный подход); 2) адатом и определенное число поверхностных атомов рассматриваются как единый кластер (молекулярный, или квантово-химический, подход). В настоящей работе мы предлагаем модель, позволяющую объединить оба подхода.

В стандартной модели адсорбции (СМА) функция Грина адчастицы, содержащей на орбитали с энергией один электрон (дырку), имеет вид

$$
G_{a}^{-1}(\omega)=\omega-\varepsilon_{a}-\Lambda(\omega)+i \Gamma(\omega) .
$$

Здесь $\omega$ - энергетическая переменная; функция уширения квазиуровня адчастицы $\Gamma(\omega)=\pi V^{2} \rho_{s}(\omega)$, где $\rho_{s}(\omega)$ - плотность состояний подложки, $V-$ усредненный по зоне Бриллюэна подложки матричный элемент взаимодействия частица-подложка; функция сдвига квазиуровня

$$
\Lambda(\omega)=V^{2} P \int_{-\infty}^{\infty} \rho_{s}\left(\omega^{\prime}\right)\left(\omega-\omega^{\prime}\right)^{-1} d \omega^{\prime},
$$

где $P-$ символ главного значения. Полюса функции Грина (1) определяются из уравнения $\omega-\varepsilon_{a}-\Lambda(\omega)=0$.
В пределе сильной связи $V^{2} \rightarrow \infty$ приближенные решения этого уравнения есть

$$
\omega_{ \pm}^{\prime} \approx\left(\varepsilon_{a} \pm \sqrt{\varepsilon_{a}^{2}+2 V^{2} N_{s}}\right) / 2
$$

где $N_{s}$ - число электронов, приходящихся на один атом подложки [7]. Полученные решения отвечают простейшему случаю двухатомной поверхностной молекулы. Ключевым моментом здесь является условие $V^{2} \rightarrow \infty$, которое следует рассматривать лишь как асимптотическое. Предлагаемая далее модель поверхностного димера (МПД) позволяет, с одной стороны, обойти эту асимптотику, а с другой - конкретизировать геометрию адсорбционного комплекса.

Разобьем пространство адсорбционной системы на две области: димер, состоящий из адсорбированной частицы и связанного с ней ковалентной связью атома подложки, и все остальное пространство. Зададим для изолированных частицы и атома подложки затравочные функции Грина в виде $g_{a}^{-1}(\omega)=\omega-\varepsilon_{a}+i s$ и $g_{s}^{-1}(\omega)=\omega-\varepsilon_{s}+i s$, где $s=0^{+}$. Исходя из уравнения Дайсона $[6,7]$ получим функции Грина для составляющих димера в виде

$$
\bar{G}_{a, s}(\omega)=\frac{g_{a, s}(\omega)}{1-g_{a}(\omega) g_{s}(\omega) t^{2}},
$$

где $t$ - энергия перехода электрона между частицей и атомом подложки. Локальные уровни, отвечающие полюсам функции Грина (2), равны

$$
\omega_{ \pm}=\left(\varepsilon_{a} \pm R\right) / 2, \quad R=\sqrt{\varepsilon_{a}^{2}+4 t^{2}},
$$

где мы положили $\varepsilon_{s}=0$. Отметим, что $\omega_{+}>0$ и $\omega_{-}<0$. Соответствующие уровням (3) локальные плотности состояний $\bar{\rho}_{a, s}(\omega)=-\pi^{-1} \operatorname{Im} \bar{G}_{a, s}(\omega)$ для частицы и атома 
равны

$$
\begin{gathered}
\bar{\rho}_{a, s}(\omega)=D_{ \pm} \delta\left(\omega-\omega_{+}\right)+D_{\mu} \delta\left(\omega-\omega_{-}\right), \\
D_{ \pm}=\left(1 \pm \varepsilon_{a} / R\right) / 2
\end{gathered}
$$

где $\delta(\ldots)$ - дельта-функция Дирака. Отметим, что состояния $\omega_{-}$и $\omega_{+}$отвечают связывающим и антисвязывающим состояниям димера, а множители $D_{-}$и $D_{+}-$ вероятностям их заполнения. Плотность состояний свободного димера $\bar{\rho}_{d i m}=\bar{\rho}_{a}(\omega)+\bar{\rho}_{s}(\omega)$ есть

$$
\bar{\rho}_{d i m}(\omega)=\delta\left(\omega-\omega_{+}\right)+\delta\left(\omega-\omega_{-}\right) .
$$

Учтем теперь взаимодействие частицы с подложкой. Легко показать, что при $\Gamma \ll t$ локальные плотности состояний (4) и (5) переходят в плотности состояний МПД вида

$$
\begin{gathered}
\rho_{a, s}(\omega)=D_{ \pm} \rho_{+}(\omega)+D_{\mu} \rho_{-}(\omega), \\
\rho_{d i m}(\omega)=\rho_{+}(\omega)+\rho_{-}(\omega), \\
\rho_{ \pm}(\omega)=\frac{1}{\pi} \frac{\Gamma(\omega)}{\left(\omega-\omega_{ \pm}-\Lambda(\omega)\right)^{2}+\Gamma^{2}(\omega)} .
\end{gathered}
$$

Задавая функции $\Gamma(\omega)$ и $\Lambda(\omega)$, будем считать, что вычленение из подложки одного атома не изменяет интегральной (средней по всей бесконечной поверхности) плотности состояний подложки.

Начнем с металлической подложки и положим $\rho_{\text {sub }}(\omega)=\rho_{m}=\mathrm{const}, \quad$ откуда получим $\quad \Gamma(\omega)=\Gamma_{m}=$ $=\pi V^{2} \rho_{m}$ и $\Lambda(\omega)=\Lambda_{m}=0$ [6,7]. Тогда плотности состояний димера $\rho_{d i m}^{m}(\omega)$ представляют собой сумму двух лоренцевых контуров: $\rho_{ \pm}(\omega)=\Gamma_{m} / \pi\left[\left(\omega-\omega_{ \pm}\right)^{2}+\Gamma_{m}^{2}\right]$. При температуре $T=0$ число заполнения димера

$$
n_{\text {dim }}^{m}=2 \int_{-\infty}^{\varepsilon_{\mathrm{F}}} \rho_{d i m}^{m}(\omega) d \omega
$$

(где $\varepsilon_{\mathrm{F}}$ - уровень Ферми системы) равно

$$
n_{d i m}^{m}=n_{+}^{m}+n_{-}^{m}, \quad n_{ \pm}^{\operatorname{dim}}=(2 / \pi) \operatorname{arccot}\left[\left(\omega_{ \pm}-\varepsilon_{\mathrm{F}}\right) / \Gamma_{m}\right] .
$$

В СМА плотность состояний, отвечающая функции Грина (1), равна $\rho_{a}(\omega)=\Gamma(\omega) / \pi\left[\left(\omega-\varepsilon_{a}-\Lambda(\omega)\right)^{2}+\Gamma^{2}(\omega)\right]$, а число заполнения адчастицы $n_{a}^{m}=(2 / \pi) \operatorname{arccot}\left[\left(\varepsilon_{a}-\right.\right.$ $\left.\left.-\varepsilon_{\mathrm{F}}\right) / \Gamma_{m}\right]$. Рассматривая величину $\Delta n^{m}=n_{d i m}^{m}-n_{a}^{m}$ при нулевой температуре, легко показать, что $\Delta n^{m}>0$, так как уровень $\omega_{-}$лежит ниже уровня $\varepsilon_{a}$.

Для описания адсорбции на полупроводнике примем для плотности состояний подложки модель Халдейна-Андерсона [7], согласно которой $\rho_{s u b}(\omega)=\rho_{s c}=\mathrm{const}$ при $|\omega|>E_{g} / 2$, и $\rho_{s}(\omega)=0$ при $|\omega| \leq E_{g} / 2$ где $E_{g}-$ ширина запрещенной зоны, центр которой принят за нуль энергии (что эквивалентно принятому нами ранее условию $\left.\varepsilon_{s}=0\right)$. Тогда $\Gamma(\omega)=\Gamma_{s c}=\pi V^{2} \rho_{s c}$ при $|\omega|>E_{g} / 2, \Gamma(\omega)=0$ при $|\omega| \leq E_{g} / 2$ и

$$
\Lambda_{s c}(\omega)=\left(\Gamma_{s c} / \pi\right) \ln \left|\left(\omega-E_{g} / 2\right) /\left(\omega+E_{g} / 2\right)\right| .
$$

Число заполнения адчастицы при нулевой температуре в МПД равно

$$
n_{d i m}^{s c}=n_{V}^{s c}+n_{l-}^{s c} \Theta\left(\varepsilon_{\mathrm{F}}-\omega_{l-}\right)+n_{l+}^{s c} \Theta\left(\varepsilon_{\mathrm{F}}-\omega_{l+}\right) .
$$

где

$$
n_{V}^{s c}=2 \int_{-\infty}^{-E_{g} / 2} \rho_{\text {dim }}^{s c}(\omega) d \omega
$$

- вклад валентной зоны полупроводника; $\rho_{d i m}^{s c}(\omega)$ дается формулами (6); $n_{l \pm}^{s c}$ - вклады локальных состояний $\omega_{l \pm}$, находящихся в запрещенной зоне; $\Theta(x)-$ функция Хэвисайда, равная единице при $x \geq 0$ и нулю при $x<0$.

Обобщая результаты, приведенные в [7], на рассматриваемую ситуацию, можем переписать выражения (7) для вкладов валентной зоны $n_{V}^{s c}$, заменив в них $\Gamma_{m}$ на $\Gamma_{s c}$ и $\omega_{ \pm}$на $\bar{\omega}_{ \pm}=\omega_{ \pm}+r$, где $r=\sqrt{\left(E_{g} / 2\right)^{2}+\Gamma_{s c} E_{g} / 2 \pi}$. Таким образом, соотношение вкладов $n_{V}^{s c}$ в МПД и CMA то же, что и для металлической подложки: $\Delta n_{V}^{s c}=n_{V}^{s c}-n_{V}>0$, где $n_{V}-$ вклад валентной зоны в СМА. С увеличением сдвига $r$ вклад валентной зоны в число заполнения уменьшается. Воспользовавшись результатом работы [8], можно утверждать также, что в областях $\bar{\omega}_{ \pm}<-E_{g} / 2$ и $\bar{\omega}_{ \pm}>\varepsilon_{\mathrm{F}}($ при $T=0)$ вкладом $n_{l \pm}^{s c}$ локальных состояний $\omega_{l \pm}^{s c}$ можно пренебречь. Поскольку энергия состояний $\omega_{l \pm}^{s c}$ определяется из уравнения (3) для $|\omega| \leq E_{g} / 2$, несложно показать, что вклад локальных состояний $n_{V}^{s c}$ в МПД может быть как больше, так и меньше значения $n_{l}$ в СМА. Действительно, пусть $\varepsilon_{\mathrm{F}}=0$ (собственный полупроводник). Тогда максимальное значение $n_{l}=2 /\left(1+2 \Gamma_{s c} / \pi E_{g}\right)$ в СМА достигается при $\omega_{l}=0^{-}$, что имеет место при $\varepsilon_{a}=0^{-}$[7]. При этом $\omega_{ \pm}= \pm t$, так что для любого конечного значения $t$ имеем $n_{d i m}^{s c}=n_{l-}^{s c}<n_{l}\left(n_{l+}^{s c}=0\right)$. Пусть теперь $\varepsilon_{a}=0^{+}$ и $n_{l}=0$ (см. (8)). Если при этом $t<E_{g} / 2$, имеем $n_{d i m}^{s c}>n_{l}=0$. Таким образом, качественный характер различия результатов для чисел заполнения в МПД и СМА определяется локализованными в запрещенной зоне полупроводника состояниями, наведенными адсорбцией.

Для описания адсорбции на однослойном графене воспользуемся упрощенной (низкоэнергетической) моделью плотности состояний графена $\rho_{g}(\omega)[7]: \rho_{g}(\omega)=2|\omega| / \xi^{2}$ при $\left|\omega_{D}\right| \leq \xi$ и 0 в остальных случаях, где $\xi-$ энергия обрезания, энергия точки Дирака $\varepsilon_{D}=0$, что в данном случае соответствует условию $\varepsilon_{s}=0$. Тогда $\Gamma_{g}(\omega)=\pi \rho_{g}(\omega) V^{2}$ и $\Lambda_{g}(\omega)=\left(2 V^{2} / \xi^{2}\right) \ln \left(\omega^{2} /\left|\xi^{2}-\omega^{2}\right|\right)$. Число заполнения адчастицы $n_{d i m}^{g}$, как и в предыдущем случае, удобно представить в виде суммы зонного $n_{b}^{g}$ и локального $n_{l-}^{g}$ вкладов (8), причем $n_{l-}^{g}=\left|1-d \Lambda_{g}(\omega) / d \omega\right|_{\omega_{l-}}^{-1}$, где $\omega_{l-}<-\xi-$ локальный уровень, лежащий ниже дна валентной зоны графена.

В [8] в рамках СМА предложены аналитические формулы, позволяющие вычислить значения $n_{b}$ и $n_{l}$. Эти формулы нетрудно переписать для МПД, приняв во внимание расщепление уровня $\varepsilon_{a}$ на состояния $\omega_{ \pm}$. Здесь 
мы, однако, поступим проще. В табл. 1 и 2 работы [8] приведены исходные данные и результаты расчета чисел заполнения адатомов щелочных металлов и галогенов. Из этих таблиц следует, что смещение уровня $\varepsilon_{a}$ в сторону меньших энергий (при переходе от щелочных металлов к галогенам) приводит к увеличению как $n_{b}$, так и $n_{l}$. Ясно поэтому, что в МПД будет наблюдаться та же тенденция, так что $\Delta n_{b, l}^{g}=n_{b, l}^{g}-n_{b, l}>0$.

Суммируя, можно утверждать, что в ситуации, когда в спектре подложки отсутствует щель (металлы, графен), использование МПД приводит к бо́льшим числам заполнения, чем СМА. Этот вывод справедлив и в том случае, если для плотности состояний металлической подложки вместо аппроксимации $\rho_{s}(\omega)=\rho_{m}=\mathrm{const}$, которая хорошо работает для простых металлов, использовать модель типа „пьедестал“ (модель Фриделя для $d$-полосы переходных металлов [9]). Однако при наличии щели в спектре графена лежащие внутри нее локальные состояния могут изменить этот вывод.

Здесь мы рассматривали $A$-тип (atop) адсорбции, когда адчастица взаимодействует с одним поверхностным атомом подложки, находясь непосредственно над этим атомом. Нетрудно, однако, обобщить полученные результаты на $B$-тип (bridge) адсорбции, когда адчастица взаимодействует с двумя ближайшими к ней соседними поверхностными атомами, и $C$-тип (center) адсорбции, когда адчастица находится в центре поверхностной элементарной ячейки. Именно эта возможность кардинально отличает МПД от СМА.

\section{Список литературы}

[1] Браун О.М., Медведев В.К. // УФН. 1989. Т. 157. № 4. C. 631-666.

[2] Dobrowski A. // Adv. Colloid Interface Sci. 2001. V. 93. N 1-3. P. 135-224

[3] Kralik M. // Chem. Papers. 2014. V. 68. N 12. P. 1625-1638. DOI: $10.2478 / \mathrm{sl} .1696-014-0624-9$

[4] Castro Neto A.H., Guinea F., Peres N.M.R., Novoselov K.S., Geim A.K. // Rev. Mod. Phys. 2009. V. 81. N 1. P. 109-162.

[5] Cooper D.R., D'Anjou B., Ghattamaneni N., Harack B., Hilke M., Horth A., Majlis N., Massicotte M., Vandsburger L., Whiteway E., Yu $V$. // International Scholarly Research Network: Condens. Matter Phys. 2012. V. 2012. P. 501686 (1-56). DOI: 10.5402/2012/501686; arXiv: 1110.6857

[6] Davison S.G., Sulston K.W. Green-function theory of chemisorption. Berlin: Springer, 2006. 211 p.

[7] Давыдов С.Ю. Теория адсорбции: метод модельных гамильтонианов. СПб.: Изд-во СПбГЭТУ „ЛЭТИ“, 2013. 235 с.; twirpx.com/file/1596114/

[8] Давыдов С.Ю., Посредник О.В. // ФТТ. 2015. Т. 57. В. 8. C. 1654-1657.

[9] Ирхин В.Ю., Ирхин Ю.П. Электронная структура, физические свойства и корреляционные эффекты в $d$ - и $f$ металлах и их соединениях. Екатеринбург: УрО РАН, 2004. $476 \mathrm{c}$. 